\title{
Finding a Crack's Position and its Parameters on the basis of Non-Destructive testing, using Eddy Currents
}

\author{
Marko Jesenik, Anton Hamler and Mladen Trlep \\ Faculty of Electrical Engineering and Computer Science \\ University of Maribor \\ Maribor, Slovenia
}

\begin{abstract}
The purpose of this paper was to find the geometry of a crack within a conductive plate and its parameters, on the basis of non-destructive testing, using eddy currents. The input data represents the measured values of magnetic flux density within the centre of the excitation coil. The position of a crack can be determined by taking into consideration any change in the magnetic flux density between the measured points. The depth and width are determined through the use of a finite-element model. These calculations are the basis for determining a function that explains how magnetic flux density changes if the depth or width has changed. After wards, through the Newton-Raphson iterative procedure using the finite-element method calculation results, the crack depth and width can be obtained. The suitability of the presented method was verified by the experimental example.
\end{abstract}

Keywords - modelling, eddy currents, finite element method, non-destructive testing

\section{INTRODUCTION}

Methods for identifying and searching for cracks using non-destructive testing has become all the more widespread and important [1]. Non-destructive testing is often used, based on considering the impact of eddy currents and the usage of different excitation coils and sensors for measuring magnetic flux density [2]. Our intention was to also determine a crack's depth and width. Some authors use analytical methods that help to solve the problem [3]. Sometimes numerical methods are used to achieve better results [4]. Our strategy is based over two steps. Firstly, calculation of the crack's length using the derivatives at each measured point towards the neighbouring points. Secondly, the determination of crack depth and width using a FEM model, combined with an iterative procedure.

\section{MEASUREMENTS}

The basis for the geometry of the test-case was the geometry presented in Fig. 1. The test-case was an aluminium plate with a crack, and an excitation coil above the plate. The coil has 566 turns and it was supplied with a sinusoidal current of $1 \mathrm{~A}$, and a frequency of $500 \mathrm{~Hz}$. Data are: $c=30 \mathrm{~mm}, a=330 \mathrm{~mm}, b=285 \mathrm{~mm}, h=7.8 \mathrm{~mm}, D_{\mathrm{i}}=$ $36.8 \mathrm{~mm}, D_{\mathrm{o}}=53 \mathrm{~mm}, h_{\mathrm{c}}=56 \mathrm{~mm}, l=40 \mathrm{~mm}, d=10 \mathrm{~mm}$ and $w=0.5 \mathrm{~mm}$. The axial hall-probe sensor was placed in the bore at the centre of the coil. The position of the coil, together with the hall-sensor, changed at each position's measurement.

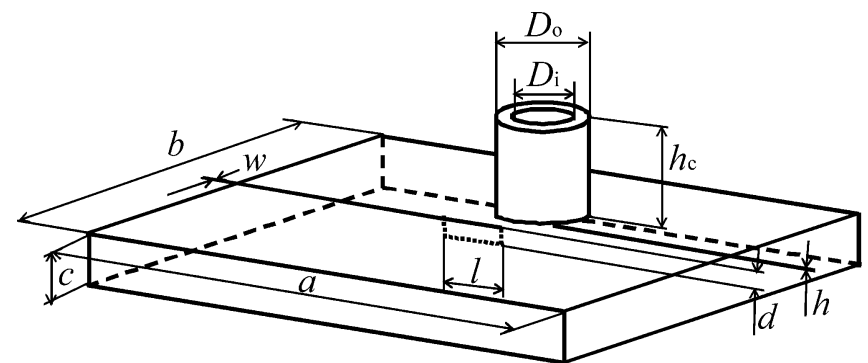

Figure 1: Plate with a crack and excitation coil.

Values obtained through the measurements, are shown in Fig. 2.

\section{DETERMINING A CRACK'S POSITION AND ITS LENGTH}

The measured $B_{\mathrm{z}}$ is exemplified with the surface, and its shape depends on the crack's parameters (length, depth, and width). Therefore, the crack's position is determined by considering any change in flux density - derivatives on the surface, as measured points. At every measured point, the derivatives are calculated in the direction of neighbouring measured points. The obtained derivatives can be 0 , positive or negative. In order to determine a crack, it is sufficient enough to know the maximum and minimum derivatives of the magnetic flux density. A crack in the material occurs when the minimum derivative is smaller than zero and the maximum is approximately equal to zero.

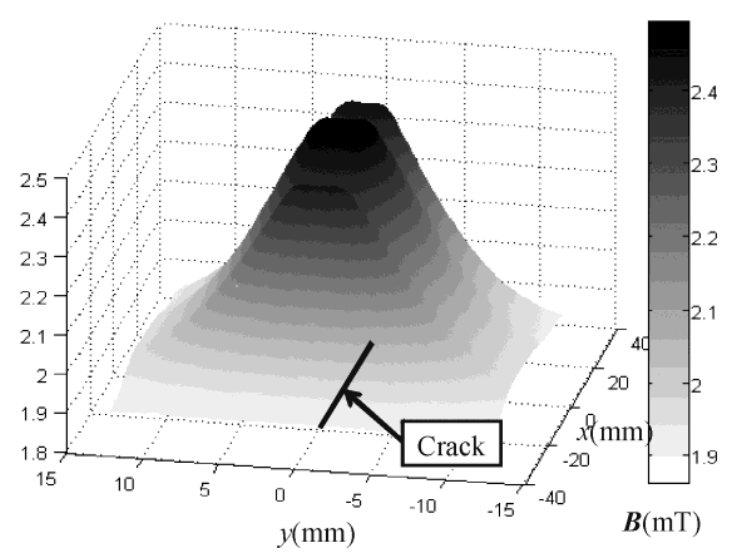

Figure 2: Measured values above the plate size $l=40 \mathrm{~mm}, d=10 \mathrm{~mm}$ and $w=0.5 \mathrm{~mm}$. 
This also occurs, when both the minimum and maximum angles are smaller than zero (this case only appears for very short cracks). A certain tolerance is taken into consideration during the calculation, since the surface of the measured values is not completely smooth. The procedure for position and length is developed on the basic of experimental testing, and searching for the universal procedure.

\section{DETERMINING THE DEPTH AND WIDTH OF A CRACK}

Depth $(d)$ and width $(w)$ influence the density course. This course above the crack can be more or less curved. The difference is mainly above and within the vicinity of a crack. Knowledge of this course, which is obtained from the measurements, makes it possible to determine $d$ and $w$. A program has been developed that connects the Finite element model (based on the $\boldsymbol{A}-V, \boldsymbol{A}$ formulation) with the Newton-Raphson iterative method. In order to determine the depth and width of a crack, it is necessary to define the function $B=\mathrm{f}(d, w)$. This function can be determined on the basis of the FEM model. In order to obtain such a function, simulations using Cedrat FLUX 3D, need to be made. Measured $B_{\mathrm{z}}$, at some points along a line in the centre of the crack, perpendicular to the crack, are the bases for the calculations of $d$ and $w$. Using the FEM calculation in FLUX 3D, the change of $B_{\mathrm{z}}$ is obtained in a single point, which depends on the $d$ and $w$, as can be seen in Fig. 3 . Points 1 to 4 are the points along a line in the centre of the crack, perpendicular to the crack. It can be concluded from the presented results in Fig. 3 , that $B_{\mathrm{z}}$ changes as the squarefunction depends on $d$ and as a linear, depends on $w$. For each point, it can be written (1).

$$
B(d, w)=a_{0}+a_{1} d+a_{2} w+a_{3} d w+a_{4} d^{2}
$$

The basis for writing $B(d, w)$ with the use of (1), is the five FEM calculations for different $d$ and $w . B(d, w)$ is written as an interpolation function, as defined in (2).

$$
\left[\begin{array}{l}
a_{0} \\
a_{1} \\
a_{2} \\
a_{3} \\
a_{4}
\end{array}\right]=\left[\begin{array}{lllll}
1 & d_{1} & w_{1} & d_{1} w_{1} & d_{1}^{2} \\
1 & d_{1} & w_{2} & d_{1} w_{2} & d_{1}^{2} \\
1 & d_{2} & w_{1} & d_{2} w_{1} & d_{2}^{2} \\
1 & d_{2} & w_{2} & d_{2} w_{2} & d_{2}^{2} \\
1 & d_{3} & w_{3} & d_{3} w_{3} & d_{3}^{2}
\end{array}\right]^{-1} \cdot\left[\begin{array}{l}
B\left(d_{1}, w_{1}\right) \\
B\left(d_{1}, w_{2}\right) \\
B\left(d_{2}, w_{1}\right) \\
B\left(d_{2}, w_{2}\right) \\
B\left(d_{3}, w_{3}\right)
\end{array}\right]
$$

Five values of $B$, calculated for differed $d$ and $w$, used to define $B(d, w)$, are shown in Fig. 4. If the value of $B$ is known in more points, we have measured values, $d$ and $w$ can be defined. Since the values of $d$ and $w$, is an important aspect in this paper, then the sum of the squares of the differences between the measured and calculated values must be the smallest. The iterative procedure leads us to the result. The Newton - Raphson iterative procedure is used.

\section{EXPERIMENTAL CXAMPLE AND CONCLUSIONS}

Analyses of a crack's parameters were made on the basis of the measured values of $B_{\mathrm{z}}$ in the coil's centre, as presented in Fig. 2.

Derivatives at each measured point are the basis when searching for the crack's-length.
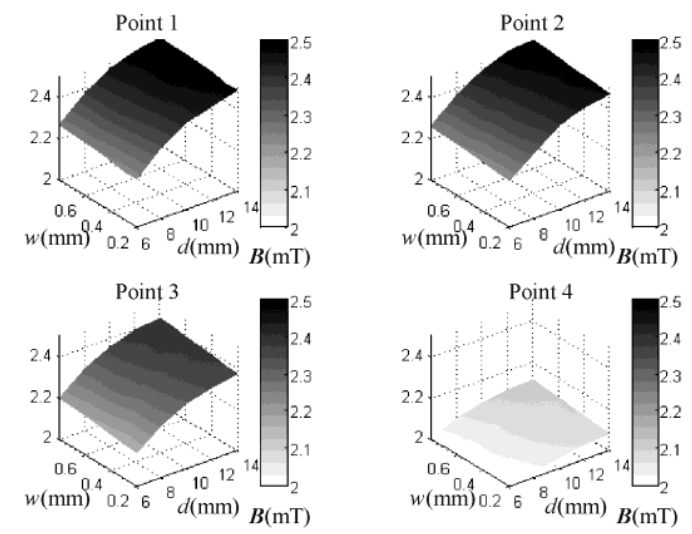

Figure 3: $B_{\mathrm{z}}$ as a function of $d(6$ to $14 \mathrm{~mm})$ and $w(0.2$ to $0.8 \mathrm{~mm})$ for points 1 to 4 .

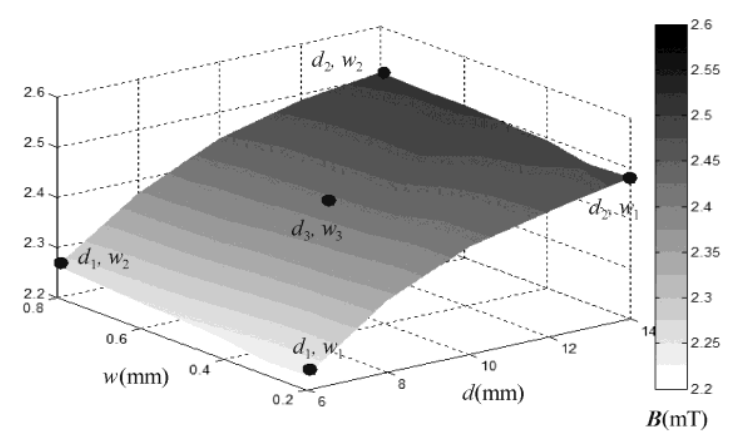

Figure 4: Five points to define $B(d, w)$ functions.

The obtained length of the crack was $39.4 \mathrm{~mm}$, which is a correct value. A 3D FEM model was created. Our own 3D FEM software was used. Five measured values of $B_{\mathrm{z}}$ were chosen for the calculations of $d$ and $w$. For each position of the measured point, five FEM calculations were made for the selected $d$ and $w$. The selected values for $d$ and $w$, as in (2), were $d_{1}=6, w_{1}=0.2, d_{2}=14, w_{2}=0.8, d_{3}=10$ and $w_{3}$ $=0.5 \mathrm{~mm}$. Five calculations at each point meant that there were 25 calculations, during which our own software was used. The Newton - Raphson iterative procedure was used for the calculations of $d$ and $w$. The iterative procedure was made until the differences between the two calculated values of $d$ and $w$ were smaller than $10^{-6} \mathrm{~mm}$. The crack-depth $d=$ $7.72 \mathrm{~mm}$ and crack-width $w=0.58 \mathrm{~mm}$ were obtained after 7 iterations. The deviation of the calculated $d$ from the real crack-depth was $23 \%$ and that of the calculated $w$ was $16 \%$. The applied procedure led us to results that are in accordance with the real crack parameters. The calculations using the FEM model were time-consuming and, as such, only five points were used to determine the $B(d, w)$ functions.

\section{REFERENCES}

[1] D. Vasić, V. Bilas and B. Šnajder, "Analyitical modelling in lowfrequency elelctromagnetic measurements of steel casing properties," NDT\&E International, vol. 40, pp. 103-111, 2007.

[2] C. Gilles-Pascaud and M. Pierantoni, "Eddy current array probe development for nondestructive testing," $16^{\text {th }}$ World Conference on NDT, Montreal, Canada, September 2004.

[3] M. Fan, P. Huang, B. Ye, D. Hou, G. Zhang and Z. Zhou, "Analytical modeling for transient probe response in pulse eddy current testing," NDT\&E International, vol. 42, pp. 376-83, 2009.

[4] G. Rubinacci, A. Tamburrino, S. Ventre, "An Efficient Numerical Model For a Magnetic Core Eddy-Current Probe," IEEE Transactions on Magnetics, vol. 44, pp. 1306-1309, 2008. 